Classification

Physics Abstracts

$06.30 \mathrm{~L}-07.50-41.10 \mathrm{D}-73.30-73.40 \mathrm{Q}$

\title{
Electrostatic forces between a metallic tip and semiconductor surfaces
}

\author{
Sylvain Hudlet, Michel Saint Jean, Bernard Roulet, Jacques Berger and Claudine \\ Guthmann
}

Groupe de Physique des Solides, Universités de Paris 7 et 6, CNRS UA17, T 23, 2 place Jussieu 75261 Paris, France

(Received July 4; accepted November 18, 1994)

\begin{abstract}
The Atomic Force Microscopies used in Resonant mode is a powerful tool to measure local surface properties: for example, the quantitative analysis of the electrical forces induced by the application of an electrical tension between a conductive microscope tip and a forwards surface in front allows the determination of the tip/surface capacitance and of the local surface work function. However, this analysis needs a well adapted model for each type of surface. In this paper, we calculate, with a simple geometrical model, the tip-surface interaction between a metallic tip and a semiconducting surface and we describe its variations with the applied tension and the tip/surface distance.
\end{abstract}

\section{Introduction.}

Atomic Force Microscopes used in Resonant mode are a powerful tool to measure local electrical surface properties. For instance, the quantitative analysis of the electrical forces induced by the application of an electrical tension between a conductive microscope tip and a forwards surface allows one to determine the local surface work function, the tip-surface capacitance and simultaneously to measure the surface topography.

In AFMR, a cantilever is excited near its resonance frequency $\Omega$, a tip being located at its end. The force gradient between the tip and the sample shifts this resonance frequency and induces a variation in the vibration amplitude of the cantilever. The resultant vibration amplitude is measured using an optical heterodyne detection and the signal is used in a feed-back loop to control the tip-surface sample distance. Topographic surface images are thus obtained at constant force gradient [1]. Moreover, by applying a modulated bias voltage $V=V_{0}+V_{1} \sin \omega t$ between the tip and the sample, added capacitive forces are applied on the tip [2]. These forces induce cantilever oscillations at $\omega$ and $2 \omega$ which depend on the electronic characteristics of the studied semiconductor surface.

To obtain the local potential with high spatial and voltage resolution $[3,4]$, we can measure 
the force $F(\omega)$ by using this new instrument. Since the attractive electrostatic force per unit area between a metallic tip and metallic surface is given by:

$$
F=\frac{Q^{2}}{2 \varepsilon_{0}}=-\frac{C_{\mathrm{I}}^{2} V^{2}}{2 \varepsilon_{0}}
$$

where $Q$ is the charge per unit area located on the tip, $V$ is the potential difference applied between the tip and the sample and $C_{\mathrm{I}}=\varepsilon_{0} / z$ is the associated capacitance per unit surface, the measurement of the oscillating force $F(\omega)$ allows to measure the local contact potentiel difference $V_{\mathrm{c}}$ between the tip and the surface. Following a procedure similar to the Kelvin method, the dc voltage $V_{0}$ is varied until the ac induced vibration of the cantilever at $\omega$, proportional to $\left(V_{0}+V_{\mathrm{c}}\right) V_{1}$, is zero. At this point, $V_{0}=-V_{\mathrm{c}}$.

On the other hand, the measurements of the variations of this force $F(\omega)$ with the tip-surface distance allows us to determine the effective curvature radius of the tip. This determination is essential to perform quantitative analysis of the experimental data. The commonly used procedure consists in measuring the electrostatic forces as a function of the tip-surface distance and to fit the experimental curve with a theoretical model, corresponding to various tip geometries [5].

In a previous paper [6], we have shown that the case of a metallic tip and a semiconductor surface is more complex than the Metal/Metal one since the charges are not located at the surface but are distributed inside the semiconductor over a distance which depends, for an applied voltage, on the tip-surface distance. The charge distribution controls the surface potentiel of the semiconductor and leads to a new variety of behaviours. In this paper, we resume the simple model which has been developped previously and present the main results. In section 2 , the calculation of the force $F(\omega)$ is presented and the different physical regimes which can be met are discussed. The calculated variations of the induced forces with the externally controlled bias voltage and the tip-surface distance are presented in section 3. We show that in the Metal/Semiconductor case a non passive capacitance has to be introduced to describe the electrical behaviour of the tip-surface system and that this capacitance induces strong effects in the $F\left(V_{0}, z\right)$ behaviour.

\section{Electrostatic force between metallic tip and semiconductor sample.}

The electrostatic force on a conducting tip held close to a $n$-doped semiconductor surface is calculated. The tip-surface system is modelized by two semi-infinite parallel plane surfaces, separated by a distance $z$ (Fig. 1), the charge distribution in the semiconductor can then be considered as one-dimensional. Moreover, to simplify the discussion we neglect the presence of an oxide at the surface and the contact potential is assumed equal to zero. The influence of these parameters on $F(\omega)$ will be discussed later.

Though very restrictive, this simple model neverthless gives a qualitative understanding of the physical situation and is an useful tool to interpretate semi-quantitatively the experimental data.

2.1 ForCE EXPRESSION. - The first step to determine the force is to calculate the total electrostatic energy per unit surface $U$ associated to the tip-surface system:

$$
U=\frac{1}{2}\left(Q_{\mathrm{M}} V_{0}+\int_{0}^{\infty} \rho(x) V(x) \mathrm{d} x\right)
$$

where $Q_{\mathrm{M}}$ and $V_{0}$ are respectively the charge (per unit surface) and the voltage on the metallic tip, $\rho(x)$ and $V(x)$ are respectively the charge density and the potential voltage inside the semiconductor (Fig. 1b). 




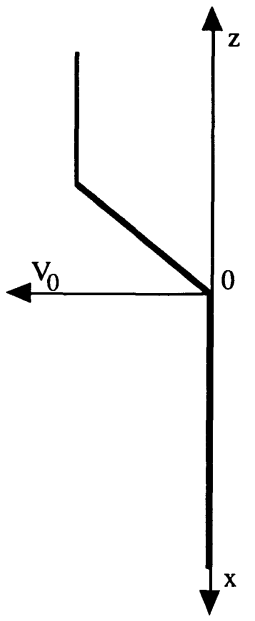

(a)

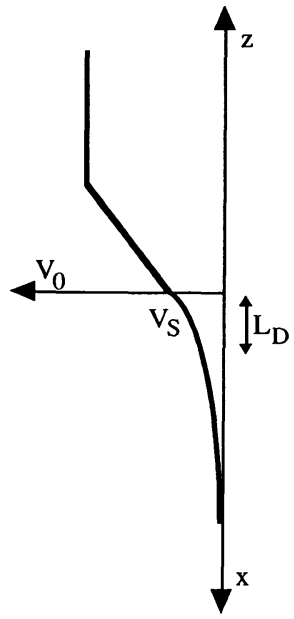

(b)

Fig. 1. - a) Metal tip/Metal surface case, b) Metal tip/Semiconductor surface. the

Introducing Poisson's equation $\nabla^{2} V=\frac{-\rho}{\varepsilon}$ and taking advantage of the value of $\nabla V$ far from semiconductor surface, $\nabla V(\infty)=0, U$ can be simply writen:

$$
U=\frac{Q_{\mathrm{M}} V_{0}}{2}+\frac{Q_{\mathrm{s}} V_{\mathrm{s}}}{2}+\frac{\varepsilon}{2} \int_{V_{\mathrm{s}}}^{0} \nabla V \mathrm{~d} V
$$

where $Q_{\mathrm{s}}=-Q_{\mathrm{M}}=\int_{0}^{\infty} \rho(x) \mathrm{d} x$ is the total charge in the semiconductor, $V_{\mathrm{s}}$ the semiconductor surface potentiel and $\varepsilon$ its dielectric constant.

Then, using the virtual work method, the force applied to the tip is equal to $-\left(\frac{\partial U}{\partial z}\right)_{Q_{\mathrm{s}}}$. As the potential voltage continuity imposes:

$$
V_{0}=V_{\mathrm{s}}-\frac{Q_{\mathrm{s}}}{C_{I}}
$$

and as $V_{\mathrm{s}}$ only depends on $Q_{\mathrm{s}}$ which is maintained constant, the applied force on the tip reduces to:

$$
F=-\frac{Q_{\mathrm{s}}^{2}}{2 \varepsilon_{0}}
$$

Notice that this expression $Q_{\mathrm{s}}$ is a complicated function of $V_{\mathrm{s}}$ which depends on the tip-surface distance and on the applied voltage (relations 4 and 10). This particular dependance explains that we cannot use a passive capacitance in the Metal/Semiconductor case.

When the applied voltage $V$ is a superposition of a constant voltage $V_{0}$ and a small modulation voltage $V_{1} \sin \omega t$, the natural procedure to obtain the explicit expression of $F(\omega)$ is to expand the force $F$ in a Taylor series and keep only the more significative terms: 


$$
F(\omega)=\frac{\partial F}{\partial V}\left(V_{0}\right) V_{1} \sin \omega t
$$

In this framework, the forces $F(\omega)$ can be expressed as function of $Q_{\mathrm{s}}$ and its derivatives with respect to $V_{\mathrm{s}}$.

$$
F(\omega)=V_{1} \sin \omega t\left(\frac{\partial F}{\partial Q_{\mathrm{s}}} \frac{\partial Q_{\mathrm{s}}}{\partial V_{\mathrm{s}}} \frac{\partial V_{\mathrm{s}}}{\partial V}\right)\left(V=V_{0}\right)
$$

In this expression, the variations of $V_{\mathrm{s}}$ with the applied voltage $V_{0}$ can be in function of the air gap and space-charge capacities, $C_{\mathrm{I}}$ and $C_{\mathrm{D}}$

$$
\left(\frac{\partial V_{\mathrm{s}}}{\partial V}\right)\left(V=V_{0}\right)=\frac{C_{\mathrm{I}}}{C_{\mathrm{I}}+C_{\mathrm{D}}} \text { with } C_{\mathrm{D}}=-\frac{\partial Q_{\mathrm{s}}}{\partial V_{\mathrm{s}}}
$$

The final expression of $F(\omega)$ is:

$$
F(\omega)=\frac{Q_{\mathrm{s}}}{\varepsilon_{0}} \frac{C_{\mathrm{I}} C_{\mathrm{D}}}{C_{\mathrm{I}}+C_{\mathrm{D}}} V_{1} \sin \omega t
$$

To evaluate this force and its variations with the external bias voltage $V_{0}$ and the tip-surface distance $z$, it is necessary to calculate $Q_{\mathrm{s}}$ and its derivative with respect to $V_{\mathrm{s}}$. In order to determine the semiconductor charge $Q_{\mathrm{s}}$ as a function of the semiconductor surface potential $V_{\mathrm{s}}$, Poisson's equation is integrated and Gauss theorem is used to connect the charge inside the semiconductor to the electric field at the semiconductor surface. In the case of $n$-doped semiconductors, the expression of $Q_{\mathrm{s}}$ versus $V_{\mathrm{s}}$ is given by:

$$
Q_{\mathrm{s}}=-\operatorname{sgn}(u) \frac{\mathrm{k} T}{q} \frac{\varepsilon}{L_{\mathrm{D}}}\left(\mathrm{e}^{u}-u-1+\frac{n_{i}^{2}}{N_{\mathrm{D}}^{2}}\left(\mathrm{e}^{-u}+u-1\right)\right)^{1 / 2}
$$

where $u=\frac{q V_{\mathrm{s}}}{k T}, L_{\mathrm{D}}=\left(\frac{\varepsilon k T}{2 N_{\mathrm{D}} q^{2}}\right)^{1 / 2}, N_{\mathrm{D}}, n_{\mathrm{i}}$ and $\varepsilon$ are respectively the dopant density, the intrinsic carrier density and the dielectric constant of the semiconductor, $q$ the elementary charge $(q>0)$.

In a second step, the continuity potential equation (4) is introduced to express the relation between $V_{0}$ and $V_{\mathrm{s}}$. Using these two equations, an unique $V_{\mathrm{s}}\left(V_{0}, z\right)$ can numerically be calculated for each set of $\left(V_{0}\right.$ and $\left.z\right)$. Ultimately, in a backforward procedure, this value is used to evaluate $Q_{\mathrm{s}}$, its derivatives and finaly the corresponding force $F(\omega)$.

2.2 Different Physical REgimes. - Before we present and discuss the variations of $F(\omega)$ with the external parameters $\left(V_{0}, z\right)$, it is convenient to keep in mind the various physical situations that can be met. According to the surface voltage $V_{\mathrm{s}}$, the surface can be populated by majority carriers (accumulation regime), minoritiy carriers (inversion regime) or can be deserted by free carriers, the unique charge staying near the surface being the ionized donors (depletion regime). These different regimes and the experimental conditions $\left(V_{0}, z\right)$ required to reach them have been previously largely discussed [6] and will be rapidly resumed in this section.

For positive surface voltage, electrons (majority carriers in $n$-doped semiconductors) are attracted to the vicinity of the air-semiconductor interface: The tip-surface system is in accumulation regime. The resulting charge distribution is essentially located near this surface and for not too small values of $z / L_{\mathrm{D}}$ (about 0.1 for $V_{0}= \pm 10 \mathrm{~V}, N_{\mathrm{D}}=10^{20}-10^{24} \mathrm{~m}^{-3}$ ), the air capacitance voltage is always larger than the semiconductor surface potential. Thus, for positive surface 
voltage, the Metal/Semiconductor system can be considered as roughly similar to a Metal/Metal system.

For negative surface voltage, we have two different regimes, according to $V_{\mathrm{s}}$ smaller or higher than $-\Phi$, where

$$
\Phi=2 \frac{k T}{q} \operatorname{Ln}\left(\frac{N_{\mathrm{D}}}{n_{\mathrm{i}}}\right)
$$

This particular voltage corresponds to the equality between the values of $-u$ and $\left(n_{\mathrm{i}} / N_{\mathrm{D}}\right)^{2} \mathrm{e}^{-u}$ in expression (10) and is reached when $z / L_{\mathrm{D}}$ verifies, for a given applied potential, the following expression:

$$
z=-\varepsilon_{0} \frac{\left(V_{0}+\Phi\right)}{Q_{\mathrm{s}}\left(-\frac{q \Phi}{k T}\right)}
$$

For small negative voltage, $-\Phi<V_{\mathrm{s}}$, the charge distribution is spread over a layer length deeper than in the accumulation regime (about $\left.\operatorname{Ln}\left(N_{\mathrm{D}} / n_{\mathrm{i}}\right) L_{\mathrm{D}}\right)$, electrons are repelled from the vicinity of the interface leaving behind a space charge region of uncompensated ionised donor ions. The tip-surface system is then in the depletion regime. In this situation, the semiconductor effect is particulary important, the tip-surface capacitance cannot be assumed to be passive (and the complete applied voltage and tip-surface dependances have to be considered).

For $V_{\mathrm{s}}<-\Phi$, the holes (minority carriers) are attracted towards a narrow region near the interface (about $\left.L_{\mathrm{D}}\right)$. The system is in the inversion regime. As the voltage magnitude $\left|V_{\mathrm{s}}\right|$ increases, a larger and larger fraction of the charge at the semiconductor surface will be hole charges and, as in the case of accumulation regime; for not too small $z / L_{\mathrm{D}}$ values, the potential decrease inside the semiconductor is negligable relative to the air capacitance contribution. Thus, for large negative surface voltage, the Metal/Semiconductor system can be roughly considered as a Metal/Metal system.

The tip-surface system reaches these different regimes according the surface voltage $V_{\mathrm{s}}$ value which depends on the $V_{0}$ and $z / L_{\mathrm{D}}$ (relations 4 and 10 ). Its variations with the applied voltage for different tip-surface distance are presented in figure 2. In these numerical calculations, the semiconductor surface is a Si- $n$ doped surface, $N_{\mathrm{D}}=10^{24} \mathrm{~m}^{-3}$ (for an other dopant concentration the main behavior of $V_{\mathbf{S}}\left(V_{0}, z\right)$ is similar). In the accumulation regime, $V_{\mathrm{s}}$ varies very slowly with $V_{0}$, and can be assumed as roughly equal to $V_{\mathrm{s} 1}(z)=2 \frac{k T}{q} \operatorname{Ln}\left(\frac{q V_{0}}{k T} \frac{\varepsilon_{0}}{\varepsilon} \frac{N_{\mathrm{D}}}{n_{\mathrm{i}}} \frac{L_{\mathrm{D}}}{z}\right)$. In contrast, the surface potential varies quickly when the system is in depletion regime and returns to a roughly constant value, equal to $V_{\mathrm{s} 2}(z)=2 \mathrm{Ln}\left|\frac{q V_{0}}{k T} \frac{\varepsilon_{0}}{\varepsilon} \frac{N_{\mathrm{D}}}{n_{\mathrm{i}}} \frac{L_{\mathrm{D}}}{z}\right|$ in the inversion regime.

Different variations of $Q_{\mathrm{s}}$, its derivatives and then for $F(\omega)$ are observed for different values of the applied voltage $V_{0}$. These various behaviours are sumarized in table $\mathrm{I}$. In particular, it is important to notice that $Q_{\mathrm{s}}$ is not always proportional to $V_{0}$ and that the simple used of a passive capacitance to describe the tip-surface system is inappropriate.

\section{Numerical evaluations of $F(\omega)$.}

In this section we present and discuss the variations of $F(\omega)$ with the bias voltage $V_{0}$ and the tip-surface distance $z$, calculated for different dopant concentrations. 


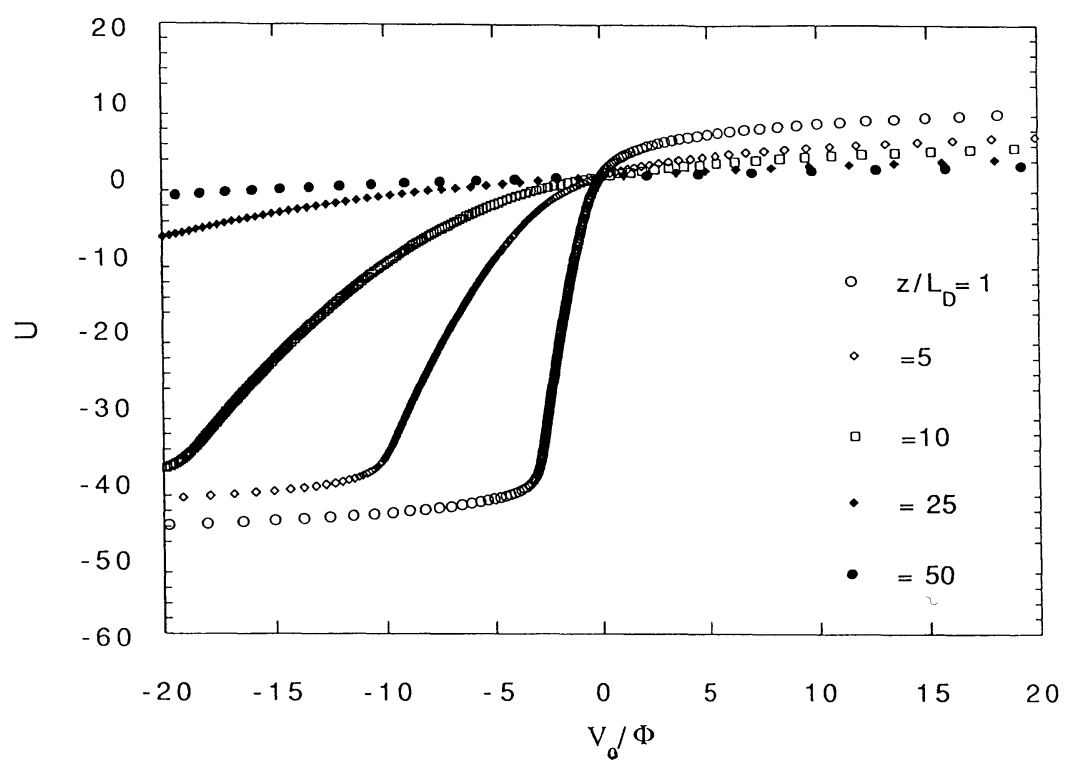

Fig. 2. - Variations of $u=q V_{\mathrm{s}} / k T$ versus $V_{0} / \Phi$ for different $z / L_{\mathrm{D}}, N_{\mathrm{D}}=10^{24} \mathrm{~m}^{-3}$. Accumulation: $u>0$, Depletion: $0>u>-40$, Inversion: $-40>u$.

3.1 VARIATIONS With APPLIED VOLTAGe $V_{0}$. - The variations of $F(\omega)$ versus $V_{0}$ for different dopant concentrations and $z=10 \mathrm{~nm}$ are shown in figure 3 , the surface being arbitrary taken equal to $10^{-14} \mathrm{~m}^{2}$. For large positive voltage, $F(\omega)$ varies linearly with $V_{0}$, the tip-surface system is in the A1 or R1 regimes (Tab. I). The different curves are parallel but shifted with respect to the metallic case by $V_{\mathrm{s} 1}(z)$. The slope of all these curves is proportional to $C_{I}^{2}$ as for a metal-metal system and is independent of the dopant concentration, in agreement with the accumulation picture. Notice that these curves go through the origin since we have neglected the contact potential. If we introduce the contact potential $V_{\mathrm{c}}$ which is similar to a modification of the potential origin, the curves will be shifted by $-V_{\mathrm{c}}$. Notice however, that for a very small voltage, the system corresponding to $N_{\mathrm{D}}=10^{20} \mathrm{~m}^{-3}$ reaches the A2 regime in which $Q_{\mathrm{s}}$ varies as $\exp \left(q V_{0} / 2 k T\right)$.

For negative voltage, the crossover from the depletion regime to the inversion regime appears on the $F(\omega)$ curves and is characterized by an elbow in $F(\omega)$ curves. More precisely, this elbow appears when the applied potential $V_{0}$ induces a surface voltage $V_{\mathrm{s}}=-\Phi$ (in the curve $N_{\mathrm{D}}=$ $10^{24} \mathrm{~m}^{-3}$, the elbow appears at $V_{0}=-9 \mathrm{~V}$, out of the figure range). Qualitatively, this behaviour can be explained as follows: In the depletion regime, the effective distance between the mobile carriers in the metal and in the semiconductor is roughly equal to $z$ plus the depletion length $L_{\mathrm{D}}$. As the magnitude of the applied voltage increases, the depletion region width increases and reaches its maximum $2 L_{\mathrm{D}} \operatorname{Ln}\left(N_{\mathrm{D}} / n_{\mathrm{i}}\right)$ when $V_{\mathrm{s}}=-\Phi[10,11]$. Then the effective capacitance decreases and the variation of $F(\omega)$ is smaller than in the Metal/Metal case. As $\left|V_{0}\right|$ increases, the system enters in the inversion region, the effective distance between the mobile carriers in the metal and in the semiconductor progressively returns to $z$.

Then, the $F(\omega)$ behaviour is again similar to the Metal/Metal case. However, in this last case, the surface voltage $V_{\mathrm{s}}$ is not zero but equal to $V_{\mathrm{s} 2}(z)$ which increases as $z$ is reduced. This effect induces shifts in the $F(\omega)$ curves. For very small voltage corresponding to the R1 regime, we can observe that $F(\omega)$ linearly varies with $V_{0}$ in a similar way than for large negative voltage for which the system is in inversion regime I1. Notice that, in this regime, these curves $F\left(V_{0}\right)$ are roughly 
Table I.

\begin{tabular}{|c|c|c|c|c|c|c|c|c|}
\hline & & $|u|<1$ & \multicolumn{2}{|c|}{ Regime $\mathbf{A} \quad \mathrm{u}>1$} & \multicolumn{2}{|c|}{ Regime I } & \multicolumn{2}{|c|}{ Regime D } \\
\hline & $\begin{array}{l}Q_{S}=-\frac{1}{\sqrt{2}} \\
\mathrm{~V}_{0}=-\mathrm{Q}\end{array}$ & $\left.+\frac{z}{\varepsilon_{0}}\right)$ & $\begin{array}{l}Q_{S}=-\frac{k T}{q} \bar{I} \\
V_{0}=\frac{k T}{q}(u\end{array}$ & $\left.\frac{\varepsilon}{\varepsilon_{0}}+\frac{z}{L_{D}} e^{u / 2}\right)$ & $\begin{array}{l}\mathrm{Q}_{S}=\frac{\mathrm{kT}}{\mathrm{q}} \frac{\varepsilon}{\mathrm{L}_{\mathrm{D}}} \\
\mathrm{V}_{0}=-\frac{\mathrm{kT}}{\mathrm{q}}(\mathrm{u}\end{array}$ & $\left.\frac{\varepsilon}{\varepsilon_{0}} \frac{\mathrm{z}}{\mathrm{L}_{\mathrm{D}}} \frac{\mathrm{n}_{1}}{\mathrm{~N}_{\mathrm{D}}} \mathrm{e}^{\mathrm{lul} / 2}\right)$ & $\begin{array}{l}Q_{s}=\frac{k T}{q} \\
V_{0}=-\frac{k}{c}\end{array}$ & $\begin{array}{l}\frac{\varepsilon}{|\mathrm{u}|} \\
\left.\frac{\varepsilon}{\varepsilon_{0}} \frac{z}{L_{D}} \sqrt{|u|}\right)\end{array}$ \\
\hline & R 1 & $\mathbf{R} 2$ & A 1 & A 2 & I1 & 12 & D1 & D2 \\
\hline$\frac{\mathrm{z}}{\mathrm{L}_{\mathrm{D}}}$ & $" 0.1$ & $<0.1$ & $>0.1$ & $" 0.1$ & $>0.1$ & $\ll 0.1$ & $\gg 0.5$ & $\ll 0.5$ \\
\hline$Q_{s}$ & $-\mathrm{C}_{\mathrm{I}} \mathrm{V}_{0}$ & $-\mathrm{C}_{\mathrm{eff}} \mathrm{V}_{0}$ & - $C_{I}\left(V_{0}-V_{S 1}\right)$ & $-\frac{k T}{q} \frac{\varepsilon}{L_{D}} e^{\mid \frac{q V_{0}}{2 k T}}$ & $-\mathrm{C}_{\mathrm{I}}\left(\mathrm{V}_{0}-\mathrm{V}_{\mathrm{S} 2}\right)$ & $\frac{k T}{q} \frac{\varepsilon}{L_{D}} e^{\mid \frac{q V_{0} \mid}{2 k T}}$ & $-\mathrm{C}_{\mathrm{I}} \mathrm{V}_{0}$ & $\sqrt{\frac{k T}{q}} \frac{\varepsilon}{L_{D}} \sqrt{\left|V_{0}\right|}$ \\
\hline$\frac{\left|F_{1}\right|}{V_{1}}$ & $\frac{C_{I}^{2}}{\varepsilon_{0}}\left|V_{0}\right|$ & $\frac{C_{\text {eff }}^{2}}{\varepsilon_{0}}\left|V_{0}\right|$ & $\frac{\mathrm{C}_{\mathrm{I}}^{2}}{\varepsilon_{0}}\left(\mathrm{~V}_{0}-\mathrm{V}_{\mathrm{S}_{1}}\right)$ & $\frac{k \mathrm{~T}}{q} \frac{\epsilon^{2}}{2 \varepsilon_{0} \mathrm{~L}^{2}} \mathrm{e}\left|\frac{\mathrm{qV} \mathrm{V}}{\mathrm{kT}}\right|$ & $\frac{\mathrm{C}_{\mathrm{I}}^{2}}{\varepsilon_{0}} I \mathrm{~V}_{0} \cdot \mathrm{V}_{\mathrm{S} 2} \mid$ & $\frac{n_{1}{ }^{2}}{N_{D}} \frac{k T}{q} \frac{\varepsilon^{2}}{2 \varepsilon_{0} L_{D}{ }^{2}} \mathrm{el}^{\mid \mathrm{q} \mathrm{V}_{0}} \mid$ & $\frac{C_{I}^{2}}{\varepsilon_{0}}\left|V_{0}\right|$ & $\frac{k T}{q} \frac{\varepsilon^{2}}{L D^{2}} \frac{1}{2 \varepsilon_{0}}$ \\
\hline
\end{tabular}

$$
\mathrm{u}=\frac{\mathrm{qV} \mathrm{S}}{\mathrm{kT}} ; \mathrm{LD}_{\mathrm{D}}=\left(\frac{\varepsilon \mathrm{kT}}{2 \mathrm{NDq}^{2}}\right)^{1 / 2} ; \quad \mathrm{V}_{0}=\mathrm{V}_{\mathrm{S}}-\frac{\mathrm{Q}_{\mathrm{S}}}{\mathrm{C}_{\mathrm{I}}} ; \mathrm{Cl}_{\mathrm{I}}=\frac{\varepsilon_{0}}{\mathrm{z}} ; \mathrm{C}_{\mathrm{eff}}=\left(\frac{\sqrt{2} \mathrm{~L}_{\mathrm{D}}}{\varepsilon}+\frac{z}{\varepsilon_{0}}\right)^{-1}
$$

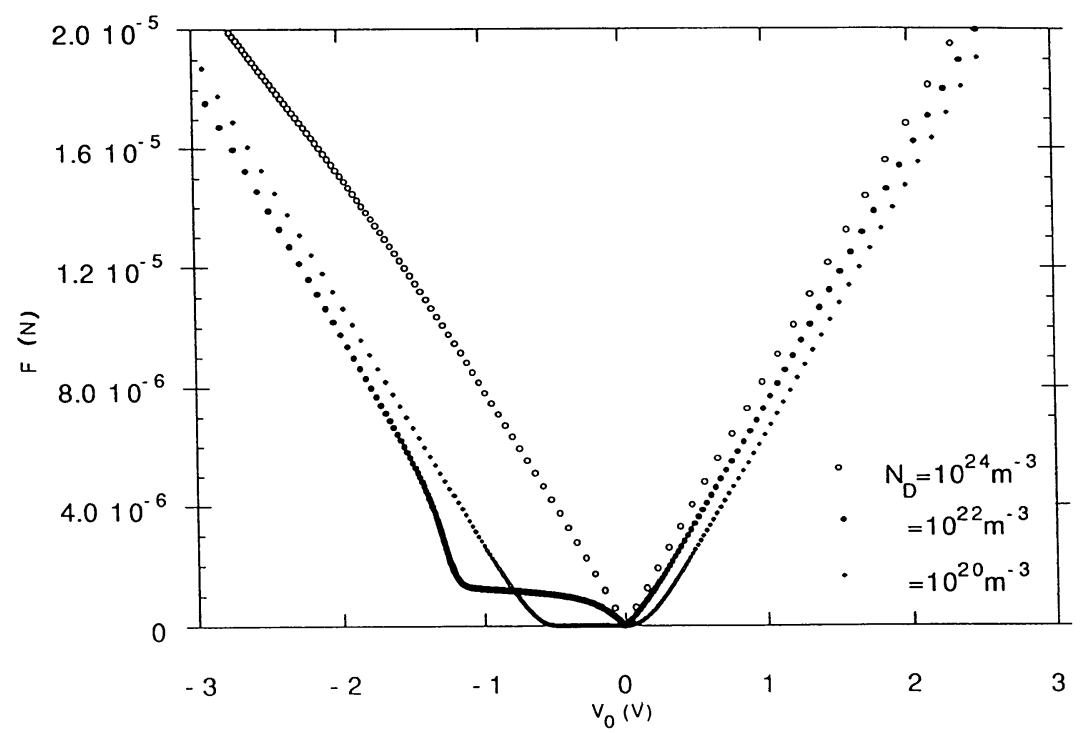

Fig. 3. - Variation of $F$ versus $V_{0}$ for different dopant concentrations, $z=10 \mathrm{~nm}$.

parallel and that their slopes $\frac{\partial F_{1}}{\partial V_{0}}\left(V_{0}=0\right)$ are proportional to $C_{\mathrm{I}}^{2}$.

Other characteristic behaviours can be observed on these curves. For instance, for $N_{\mathrm{D}}=$ $10^{22} \mathrm{~m}^{-3}$ and for $V_{0}$ about $-1.8 \mathrm{~V}$, a tip-surface distance equal to $10 \mathrm{~nm}$ corresponds to a regime very close to the inversion regime $\mathrm{I} 2$ in which $F(\omega)$ varies as $\exp q\left|V_{0}\right| / k T$. For smaller voltage, 


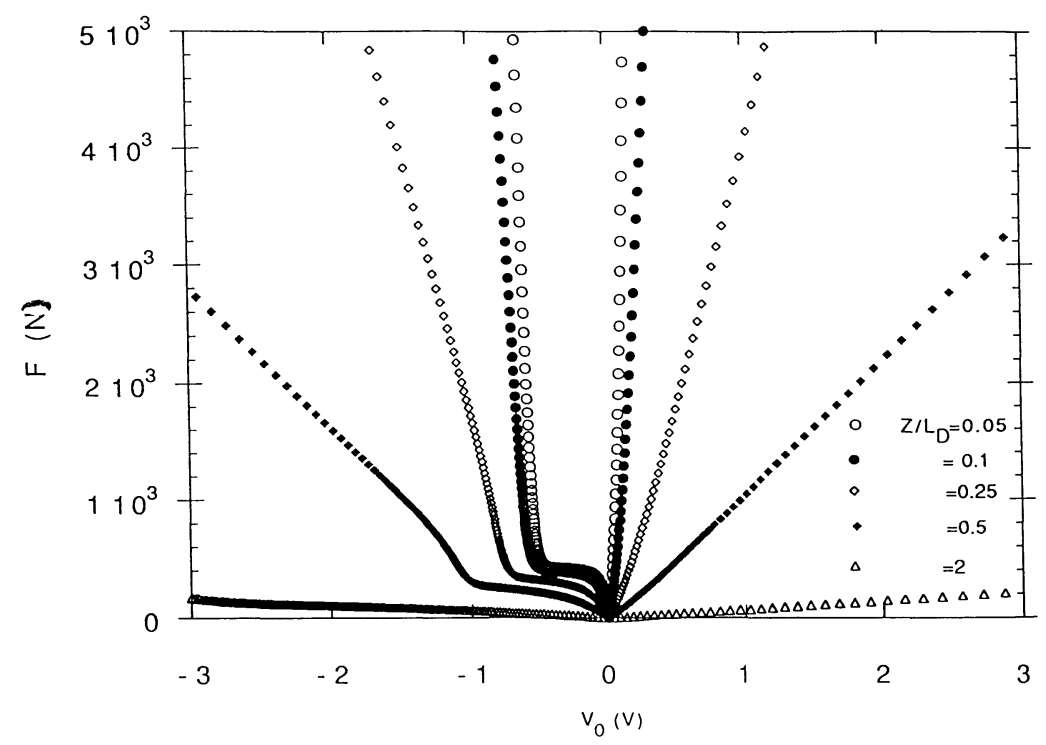

Fig. 4. - Variation of $F$ versus $V_{0}$ for different $z / L_{\mathrm{D}}, N_{\mathrm{D}}=10^{20} \mathrm{~m}^{-3}$.

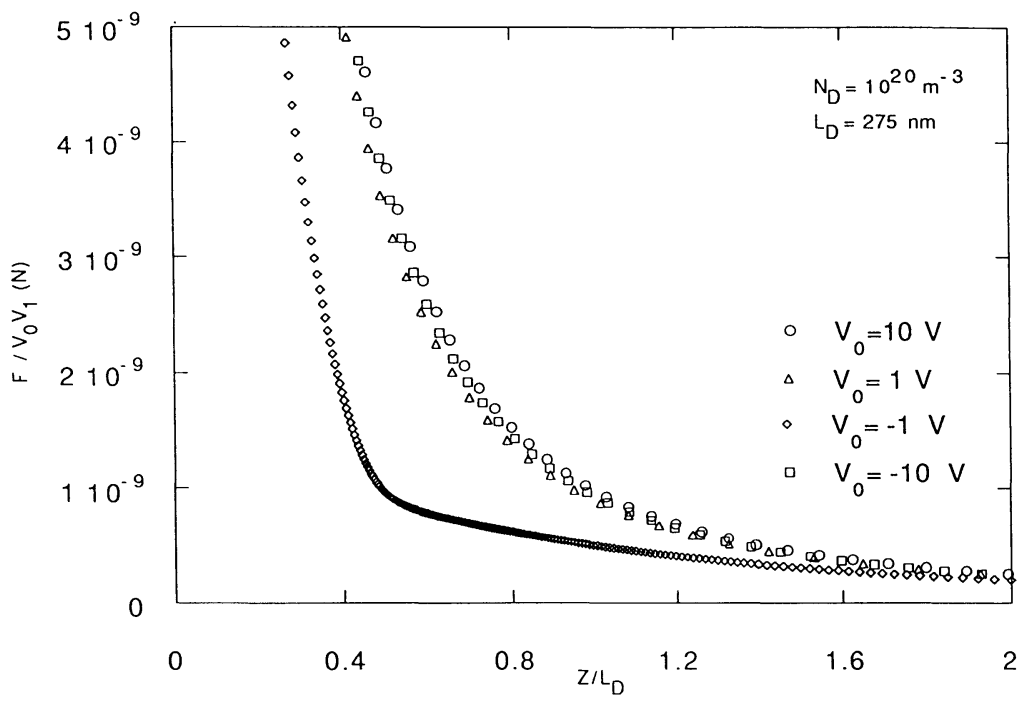

Fig. 5. - Variation of $F$ versus $z / L_{\mathrm{D}}$ for different applied voltage, $N_{\mathrm{D}}=10^{20} \mathrm{~m}^{-3}$.

the system is in the D2 depletion regime and $F(\omega)$ correspond to a roughly constant value.

3.2 InfluenCE OF THE TIP-SURFACE DISTANCE. - The variations of $F(\omega)$ versus $V_{0}$, for different values of $z / L_{\mathrm{D}}, N_{\mathrm{D}}=10^{20} \mathrm{~m}^{-3}$, are shown in figure 4 . It is interesting to observe that the variation of the elbow position with $z / L_{\mathrm{D}}$ is very sensitive to the tip-surface distance, its value decreasing with a decrease of the tip-surface distance $z$.

Moreover, different behaviours of $F\left(V_{0}, z\right)$ versus $z$ can be observed. For instance, for $V_{0}=$ $-0.6 \mathrm{~V}$, we can follow very precisely the different regimes crossed when the tip-surface distance 
increases. For $z / L_{\mathrm{D}}=0.05: F(\omega)$ increases exponentially in the inversion regime $\left(V_{\mathrm{s}}<-\Phi\right)$ and is constant for the depletion regime $\left(V_{\mathrm{s}}>-\phi\right)$. As $z / L_{\mathrm{D}}$ increases, the system reaches the depletion regime $\mathrm{D} 2$ for $z / L_{\mathrm{D}}=0.25$, and the regime $\mathrm{D} 1$ for $z / L_{\mathrm{D}}>0.5$. In the same time, the elbow shifts towards the large negative voltage.

The main consequence of these semiconductor effects is to modify drastically the shape of the curves describing the variations of $F(\omega)$ with $z / L_{\mathrm{D}}$. For instance, figure 5 shows the variations of $F(\omega) / V_{0} V_{1}$ versus $z / L_{\mathrm{D}}$ for different values of $V_{0}$, the dopant concentration being fixed to $10^{20} \mathrm{~m}^{-3}$. The curves corresponding to an applied potential $V_{0}= \pm 10 \mathrm{~V}$ and $1 \mathrm{~V}$ show the same variations and are similar to those obtained for an Metal/Metal system. In contrast the curve corresponding to $V_{0}=-1 \mathrm{~V}$ presents a very different behaviour: a strong change in the curve slope can be observed which appears for $z / L_{\mathrm{D}}$ about $0.5(z=140 \mathrm{~nm})$. This effect corresponds to the crossover between depletion and inversion regimes.

We want to emphasize that it is essential to keep in mind that this feature in the $F(\omega)$ curves cannot be analyzed using only geometric considerations, and that the Metal/Semiconductor system specificity has to be integrated in the interpretations of such experimental results.

\section{Conclusion.}

In this study, we have calculated the capacitive force $F(\omega)$ between the metallic tip of an AFMR microscope and a $n$-doped semiconductor surface separated by an air gap and submitted to a modulated applied voltage. The variations of these forces versus the static applied voltage and the tip-surface distance have been investigated. This study shows that, in this system, the force variations present specific behaviours wich are not observed when the surface is metallic. Different regimes are exhibited according to the values of the tip-surface distance and the static applied voltage.

These various regimes originate from the different behaviours of the semiconductor surface charge $Q_{\mathrm{s}}$ which depends on the applied voltage $V_{0}$ and on the relative importance of the air gap contribution with respect to the potential decrease inside the semiconductor, characterised by the Debye length $L_{\mathrm{D}}$.

Roughly, we can conclude that for a fixed applied voltage, when the tip-surface distance $z$ is much higher than $L_{\mathrm{D}}$ the system is roughly similar to the Metal/Metal case. In contrast when $z$ is smaller than $L_{\mathrm{D}}$, this simple model is not adapted and some features appear in the curves representing the variations of the forces versus the applied voltage.

Another aspect of these semiconductor effects is reflected in the variations of $F(\omega)$ with the tip-surface distance. In particular we have shown that the determination of the tip-surface capacitance geometrical characteristics by fitting the experimental curves is not allowed in all the experimental cases. To be valid, the procedure requires high applied voltages such as $\left|V_{\mathrm{s}}\right|>\Phi$.

Moreover, various physical quantities can be extracted from these presented curves. The contact potential $V_{\mathrm{c}}$ between the tip and the surface can be obtained from the measurement of the voltage corresponding to $F(\omega)=0$. In this situation, the applied potential $V_{0}$ is exactly equal to $-V_{c}$. Using a feed back loop asserving the applied voltage to fix $F(\omega)=0$, image of iso-contact potential can be obtained. The local dopant concentration can also be determined. For small $z / L_{\mathrm{D}}$, we have shown that, in this case, the Depletion/Inversion transition is characterized by an elbow in the $F(\omega)$ curves. Since, this feature appears when $V_{\mathrm{s}}=-\Phi$, we can extract the dopant concentration from the measurements of the voltage position of this elbow.

To conclude, notice that this study presents some limits. in our calculation, we have neglected the presence of an oxide layer on the semiconductor surface. However, this assumption does not qualitatively modify the present results, since, its presence is in fact qualitatively similar to an effective reduction of the tip-surface distance with respect to the case without oxide. Moreover, 
in this model, the tip and the semiconductor surfaces are assumed to be parallel plates. This geometry does not correspond to the experimental configuration when the tip-surface distance is smaller than the tip curvature radius $(z<100 \mathrm{~nm})$; in this case the tip is better described by an ellipsoid electrode. Neverthless this simple model, which can be extended to an ellipsoid/plate geometry, offers the opportunity to give useful semiquantitative understanding of the electrostatic force which are accounted in the AFMR.

\section{References}

[1] Martin Y., Abraham D.W., Wickramasinghe H.K., Appl. Phys. Lett. 52 (1988) 1103.

[2] Terris B.D., Stern J.E., Rugar D., Mamin H.J., Phys. Rev. Lett. 63 (1989) 2669.

[3] Nonnenmacher M., O’Boyle M., Wickramasinghe H.K., Ultramicroscopy $42-44$ (1992) 268.

[4] Weaver J.M.R., Abraham D.W., J. Vac. Sci. Technol. B9 (1991) 1559.

[5] Huang Wen Hao, Baro A.M., Saenz J.J., J. Vac. Sci. Technol. B9 (1991) 1323.

[6] Hudlet S., Saint Jean M., Roulet B., Berger J., Guthmann C., J. Phys. France 4 (1994) 1725-1742.

[7] Sze S., The physics of Semiconductor Devices (Wiley, New York, 1981). 